\title{
Michael Meaney: Wie die Zuwendung der Eltern die Stressvulnerabilität beeinflusst: Molekularbiologische Grundlagen sozialer Erfahrung
}

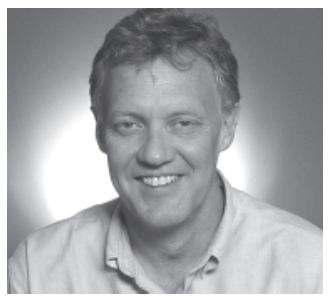

\begin{abstract}
Dr. Micheal Meaney ist Professor an den Abteilungen für Psychiatrie sowie für Neurologie and Neurochirurgie der McGill Universität, Montreal, und Leiter des dortigen Forschungsprogramms "Verhalten, Gene und Umwelt». Zudem ist er Forschungsdirektor am Douglas Hospital Research Center, ebenfalls Montreal, Kanada. Schwerpunkte seiner wissenschaftlichen Arbeit sind die biologischen Mechanismen, durch die sich elterliches Pflegeverhalten und Umweltbedingungen auf die Aktivität von Genen auswirken, welche die verhaltensmäßige und neuroendokrine Stressreaktivität regulieren. Er hat über 180 wissenschaftliche Arbeiten veröffentlicht und zahlreiche Preise für seine Untersuchungen im Zusammenhang mit Stress erhalten. Das Interview wurde von Prof. Dr. Dirk Hellhammer, Trier, für VerHALTENSTHERAPIE geführt und von Sabine Albertz-Habscheid übersetzt.
\end{abstract}

Bereits im neunzehnten Jahrhundert äußerte Sigmund Freud im «Entwurf einer Psychologie» die Hoffnung, dass eines Tages die spezifischen Hirnfunktionen bekannt würden, über welche psychische und soziale Erfahrungen eine erhöhte Anfälligkeit für Krankheiten vermitteln. Nach mehr als hundert Jahren intensiver Forschung ist dies nun erstmals exemplarisch gelungen. Ihre Forschung hat diesen Durchbruch in eine neue Ära der Verhaltensforschung ermöglicht. Sie wurden als Klinischer Psychologe ausgebildet. Erzählen Sie uns doch, wie Sie wissenschaftlich vorgegangen sind.

Michael Meaney: Nun, zunächst musste die Art des Zusammenhangs zwischen elterlicher Pflege und deren Folgen für die Entwicklung definiert werden. Anschließend galt es, die Mechanismen aufzuklären, welche diese lebenslang anhaltenden Effekte auslösen. Bei Ratten werden Ängstlichkeit und Stressvulnerabilität weitgehend durch die Intensität mütterlicher Brutpflege (licking and grooming) begünstigt, wobei verstärktes «Licking» eine abgedämpfte Stressreaktion in Bezug auf beide Parameter erzeugt. Die erste Herausforderung war die Beschreibung der Mechanismen bzw. der biologischen Prozesse, die durch mütterliche Pflege modifiziert werden können. Wir konzentrierten uns auf die HypophysenHypothalamus-Nebennierenrinden-Achse (HHNA). Die HHNA ist eine der Hauptachsen, über welche das Gehirn Körperfunktionen steuert. Im Hypothalamus wird Corticotropin Releasing Hormone (CRH) freigesetzt, was die Hypophy- se und die Nebennieren veranlasst, das Stresshormon Cortisol zu produzieren.

Die Aktivität der HHNA wird über eine inhibierende Rückkopplungsschleife kontrolliert. Hier sind vor allem die Glukokortikoide zu nennen, die auf ganz unterschiedliche Gehirnstrukturen Einfluss nehmen, um die HHNA zu regulieren. Die Wirkung der Glukokortikoide wird im Gehirn über spezifische Rezeptoren vermittelt. Das bedeutet, dass das Steroidhormon mit einem Rezeptorprotein interagiert, und zwar auf ganz unterschiedlichen Ebenen im Gehirn. Der Hippokampus ist dabei die Hirnregion, die in erster Linie mit diesem negativen Feedbackeffekt in Verbindung gebracht wurde. Wie man sich vorstellen kann, ist hier natürlich die Anzahl der Glukokortikoid-Rezeptorproteine im Gehirn von entscheidender Bedeutung. Das heißt, dass eine erhöhte Anzahl der Glukokortikoid-Rezeptoren im Hippokampus diese Struktur sensitiver für die dort zirkulierenden Glukokortikoide macht und damit die Wirkung des Glukokortikoid-Feedbacks verstärkt.

Dies bedeutet beispielsweise, dass eine erhöhte Cortisolfreisetzung bei Stress über Rezeptoren im Hippokampus die HHNA so lange hemmt, bis sich die Werte wieder normalisiert haben?

Michael Meaney: Zumindest gilt das für unser Tiermodell. So konnten wir zeigen, dass Ratten mit erhöhter Anzahl von Glukokortikoid-Rezeptoren im Hippokampus eine verringer-

\begin{tabular}{ll}
\hline KARGER & ( ) 2005 S. Karger GmbH, Freiburg \\
Fax +49 7614 520714 & Accessible online at: \\
$\begin{array}{l}\text { E-mail Information@Karger.de } \\
\text { www.karger.com }\end{array}$ & www.karger.com/ver
\end{tabular}


te Stressantwort zeigen. Bei Ratten legt «maternal licking» während der ersten Lebenswoche die Menge der Glukokortikoid-Rezeptorproduktion im Hippokampus fest. Rattenjunge, die in der ersten Lebenswoche häufiger gepflegt werden, zeigen erhöhte Glukokortikoid-Rezeptorspiegel im Hippokampus und eine verringerte HHNA-Antwort auf Stress im Vergleich zu Rattenjungen, die weniger häufig von der Mutter gepflegt wurden. Mütterliche Pflege resultiert also in einer Erhöhung der hippokampalen Glukokortikoid-Rezeptoren. Dies wird vermittelt über die Interaktion der durch «licking» ausgelösten neuronalen Veränderung mit dem Gen, das für den Glukokortikoid-Rezeptor kodiert.

\section{Wie kann man sich diese Interaktion vorstellen?}

Michael Meaney: Diese neuronalen Veränderungen modifizieren die chemischen Eigenschaften der Desoxyribonukleinsäure (DNS/DNA) die den Glukokortikoid-Rezeptor kodiert. Dies geschieht über einen klassischen epigenetischen Mechanismus. Wir sprechen hier natürlich nicht über Veränderungen in der Gensequenz. Die Gensequenz, also die Abfolge der Basenpaare, bleibt unangetastet von mütterlicher Pflege oder anderen Umweltvariablen, die wir betrachten. Also, nicht die Gensequenz, sondern die Genaktivität wird verändert. Dieser Prozess spielt sich in einer Region des Gens ab, die als Promoterregion bekannt ist. Der Promoter ist nicht an der Proteinkodierung beteiligt, sondern er ist zuständig für die Regulation der Transkription des Gens. Das heißt, der Promoter steuert die Genaktivität und beeinflusst somit die Menge an Protein, die produziert wird.

Mütterliches «licking and grooming» erzeugt das, was wir «strukturelle Veränderung eines einzigen Nukleotids» nennen. Ein Cytosin innerhalb der Promoterregion des Glukokortikoid-Rezeptors wird modifiziert. Diese strukturelle Modifikation besteht ganz einfach aus dem Anfügen oder Weglassen einer Methylgruppe am Cytosin. Wenn eine Methylgruppe angelagert ist, bleibt der Promoter größtenteils inaktiv, wodurch die Glukokortikoid-Rezeptorproduktion vermindert wird. Bleibt das einzelne Cytosin-Nukleotid unmethyliert, so ist die Promoterregion aktiv und es werden mehr Glukokortikoid-Rezeptoren gebildet. Mütterliche Pflege modifiziert den Methylierungsstatus dieses einzelnen Cytosins, wodurch die Glukokortikoid-Rezeptorproduktion innerhalb des Hippokampus verändert wird. Auf diesem Wege beeinflusst mütterliches Pflegeverhalten die HHNA-Responsivität ein Leben lang.

\section{Könnte dies auch Konsequenzen für innovative Ansätze in} Psychotherapie und Pharmakotherapie haben?

Michael Meaney: Man ist schon länger davon ausgegangen, dass die Unterschiede in der Methylierung der DNA von Umweltereignissen ausgelöst werden. Allerdings glaubte man, dass sich diese Einflüsse sehr früh im Leben auswirken, in der Regel nur während der Embryonalentwicklung, und dass diese Modifikation irreversibel festlegt, ob ein DNA-Abschnitt methyliert wird oder nicht. Wie sich nun durch neueste Studien unserer Forschergruppe zeigt, ist der Methylierungsstatus auch später noch umkehrbar. In der Tat wurde der ursächliche Zusammenhang zwischen unterschiedlicher Methylierung des Promoters und Unterschieden in der Stressantwort experimentell nachgewiesen. Entfernt man durch pharmakologische Intervention bei einem Nachkommen einer «low-licking» Rattenmutter dauerhaft die Methylgruppe im Promoter, so hat dies einen Anstieg der GlukokortikoidRezeptoren zur Folge, womit er dann phänotypisch einem Nachkommen einer «high-licking» Mutter ähnelt und sich seine Stressreaktion wieder normalisiert.

Nachdem nun klar war, dass diese Modifikationen reversibel sind, ist die nächste Frage: Welche therapeutischen Interventionen sind notwendig und ausreichend, um eine langfristige Veränderung der Methylierung hervorzurufen? Leider kennen wir die Antwort hierauf noch nicht. Wir vermuten allerdings, dass es notwenig sein wird, das Milieu innerhalb des Hippokampus, wahrscheinlich über eine längere Zeitspanne, so zu verändern, das die Methylgruppe am betreffenden DNA-Abschnitt längerfristig entfernt wird.

Welches könnten die relevanten Stimuli sein, die solche Effekte hervorrufen?

Michael Meaney: Natürlich kann es sich um eine pharmakologische Manipulation handeln. Aber theoretisch besteht kein Grund zu der Annahme, dass tatsächlich nur eine pharmakologische Intervention hilfreich sein kann. Die Manipulation könnte auch in einer Anreicherung positiver Umwelteinflüsse bestehen, oder es könnte sich ebenso wahrscheinlich um eine psychotherapeutische Intervention handeln. Leider wird sich das so lange nicht klären, bis der genaue Mechanismus bekannt ist, welcher dem Methylierungsstatus unterliegt. Aber ich bin optimistisch, dass wir das bald wissen.

Ihre Forschung konnte nun zum ersten Mal zeigen, dass es nicht mehr unwahrscheinlich ist, Freuds Traum zu verwirklichen, das heißt die biologischen Gegebenheiten zu verstehen, über die psychosoziale Erfahrungen zu molekularen Veränderungen führen. Was ist Ihre Vision für die Zukunft der Verhaltensmedizin?

Michael Meaney: Das soziale Umfeld scheint ein außerordentlich wirksamer Regulator biologischer Systeme zu sein. Dies zeigt sich bis hin zur Ebene der DNA, die in ihrer Funktion sozialer Regulation unterliegt. Wenn also die Aktivität der DNA über den gesamten Entwicklungszeitraum sozial bestimmt wird, sollte das auch bedeuten, dass sie durch soziale und psychologische Interventionen modifiziert werden kann. Und es gibt keinen Grund anzunehmen, dass diese Effekte nur auf Vorgänge im Gehirn beschränkt sein sollten.

Veränderungen im Gehirn können auch Änderungen der Im- 
munfunktion und Veränderungen des endokrinen Systems bewirken sowie bei Signalveränderungen relevant werden, die bei kardiovaskulären Erkrankungen, Diabetes usw. bekannt sind. Wir werden letztendlich vielleicht erkennen, dass soziale Interventionen therapeutische Effekte hervorrufen können, vermittelt über ein weites Spektrum an Veränderungen bis hin zu strukturellen Modifikationen auf Ebene der DNA. Und dies kann in der Tat bei einer großen Anzahl von Erkrankungen von Bedeutung sein.

Sie sind in Montreal geboren und haben eine großen Teil Ihres wissenschaftlichen Lebens hier verbracht. Montreal war ebenfalls die Heimat von Hans Selye, Walter Penfield und Donald Hebb. Hat dies Ihr Interesse an Stressforschung beeinflusst?

Michael Meaney: Hundertprozentig! Ich denke in der Tat, dass sich meine Forschung nahezu vollständig hieraus ableitet und dass ihr Wert an der Bedeutung dieser Persönlichkeiten liegt, von denen diese Forschungsideen abgeleitet sind. Ich denke, dass die Forschung, die wir hier durchführen, durchaus den Einfluss von Hebb widerspiegelt, indem sie den Einfluss von Umwelt auf neuronale Funktionen und biologische Ereignisse fokussiert. Aber ebenso die Bedeutung von Stress und stresssensitiven Systemen für die Krankheitsentwicklung, wie sie von Hans Selye beschrieben wurde.

Ich denke daher, wenn man sieht, dass jemand aus Montreal kommt, und wenn dieser dann auch noch die Rolle von Umwelteinflüssen bei individuellen Unterschieden der Stressantwort untersucht, dann liegt es auf der Hand, dass sein Interesse durch frühere Erfahrungen mit Hebb, Selye und deren Studenten geprägt wurde.

Gibt es für klinische Psychologen Möglichkeiten, sich mit diesem Forschungsgebiet vertraut zu machen?

Michael Meaney: Vom 24.-27. September 2005 richten wir in Montreal das «36th Annual Meeting of the International Society of Psychoneuroendocrinology» (ISPNE) aus. Hauptthema des Kongresses werden die Untersuchungen zum Verhältnis von sozioökonomischem Umfeld und Gesundheit in Bezug auf psychoneuroendokrinologische Faktoren sein. Die epidemiologische Forschung hat ganz klar die Bedeutung des sozioökonomischen Status in Zusammenhang mit Gesundheit dargelegt. Die Herausforderung besteht nun darin, die Mechanismen zu verstehen, durch die diese sozialen und ökonomischen Variablen auf unsere Gesundheit Einfluss nehmen. Wir haben zumindest teilweise versucht, den Kongress um dieses Thema herum zu gestalten.

Wir danken für das Gespräch und wünschen Ihnen weiterhin viel Erfolg bei Ihrer hoch interessanten Forschung. 